BULL. AUSTRAL. MATH. SOC.

VOL. $20(1979), 17-34$.

\title{
Indecomposable representations of certain commutative quivers
}

\section{P.W. Donovan and M.-R. Freislich}

\begin{abstract}
This paper gives a detailed classification of the indecomposable representations of one non-trivial "commutative quiver", gives brief details of certain generalisations, and uses methods likely to be more widely applicable.
\end{abstract}

Throughout this paper $K$ denotes a field whilst all modules are left modules of finite dimension over $K$. The indecomposable modules are classified for the two $K$-algebras whose presentations follow. In both cases $\left\{\varepsilon_{1}, \varepsilon_{2}, \varepsilon_{3}, \varepsilon_{4}, \varepsilon_{5}, \varepsilon_{6}\right\}$ denotes a complete set of primitive orthogonal idempotents whilst a generator $\psi_{i j}$ is understood to satisfy the relationship $\psi_{i j}=\varepsilon_{i} \psi_{i j} \varepsilon_{j}$ :

$$
\begin{array}{r}
R_{1}=K\left\langle\varepsilon_{1}, \varepsilon_{2}, \varepsilon_{3}, \varepsilon_{4}, \varepsilon_{5}, \varepsilon_{6}, \gamma_{51}, \gamma_{52}, \gamma_{53}, \gamma_{54}, \delta_{16}, \delta_{26}, \delta_{36}, \delta_{46}\right| \\
\left.\gamma_{51} \delta_{16}=\gamma_{52} \delta_{26}, \gamma_{53} \delta_{36}=\gamma_{54} \delta_{46}\right), \\
R_{2}=K\left\langle\varepsilon_{1}, \varepsilon_{2}, \varepsilon_{3}, \varepsilon_{4}, \varepsilon_{5}, \varepsilon_{6}, \gamma_{15}, \gamma_{25}, \gamma_{35}, \gamma_{45}, \alpha_{56}, \beta_{56}\right| \\
\left.\gamma_{15} \alpha_{56}=\gamma_{25} \alpha_{56}=\gamma_{35} \beta_{56}=\gamma_{45} \beta_{56}=0\right) .
\end{array}
$$

These algebras are best understood as quiver algebras in the sense of [3]. For example, a $R_{1}$-module $M$ is just a sextuple of vector spaces $M_{1}\left(=\varepsilon_{1} M\right), \ldots, M_{6}\left(=\varepsilon_{6} M\right)$ together with the eight linear mappings $\gamma_{5 i}: M_{i} \rightarrow M_{5}, \delta_{i 6}: M_{6} \rightarrow M_{i}$ satisfying the relations shown above. The

Received 27 October 1978. Both authors are greatly indebted to M.C.R. Butler and S. Brenner for much assistance in many aspects of this paper. 
significance of these algebras lies in the fact that their representation theory is quite complex but still tame in the sense of [3]. This will be proved in this paper. It will be noted that in the above interpretation of $R_{1}$-modules the special cases

$$
\begin{aligned}
& \text { (1) } M_{5}=0 \text { and } \\
& \text { (2) } M_{6}=0
\end{aligned}
$$

correspond to the non-trivial '4-subspace problem' solved in [2], [5], and elsewhere. Likewise the classification of $R_{2}$-modules with

(3) $M_{6}=0$ and

(4) $M_{1}=M_{2}=M_{3}=M_{4}=0$

correspond to different 'extended Dynkin diagram' problems, to wit the 14-subspace problem and the 'Kronecker' problem. In Section 7 the class of problems of this type accessible to the methods of this paper is discussed.

Section 1 contains a discussion of two functors, the first of which establishes a virtual equivalence between the above two classification problems and the second of which simplifies the latter problem. Section 2 briefly describes the matrix regress method of Nazarova and Roiter which is used in Sections 4 and 5 . Section 3 gives the classification of indecomposable 4-subspace systems. Section 4 gives a direct determination of the indecomposable $R_{2}$-modules whilst Section 5 gives an alternative approach more suitable for the construction of canonical forms. The quadratic form phenomenon of [3] is discussed in section 6 .

\section{The functors used in the classification}

The contents of this section are special cases of work of Auslander, Platzeck, Reiten [1], which has recently been extended by Butler. The functors discussed below both have evident adjoints which may be used to give direct proofs of the properties mentioned.

The functor $\Psi: \bmod \left(R_{1}\right) \rightarrow \bmod \left(R_{2}\right)$ is defined as follows, using the notation of the introduction. If $M \in \bmod \left(R_{1}\right),(\Psi M)_{i}=i_{i}$ for $i \neq 5$, 
$(\Psi M)_{5}=\operatorname{ker}\left(M_{1} \perp_{2}{ }_{2} \perp_{3} M_{4} M_{4}\right) \rightarrow M_{5} ;$ the new $\gamma$ mappings are the projections and $\alpha_{56}\left(m_{6}\right)=\left(0,0, \delta_{36} m_{6},-\delta_{46} m_{6}\right)$, $\beta_{56}\left(m_{6}\right)=\left(\delta_{16} m_{6}, \delta_{26} m_{6}, 0,0\right)$ for $m_{6} \in M_{6}$. It establishes a bijection between the full subcategory of $\bmod \left(R_{1}\right)$ whose objects are the $R_{1}$-modules without direct summands isomorphic to the projective module $R_{1} \varepsilon_{5}$, and a full subcategory of $\bmod \left(R_{2}\right)$.

The functor $\Phi: \bmod \left(R_{2}\right) \rightarrow \bmod \left(R_{2}\right)$ takes $M$ to $M^{\prime}$ where $M_{i}^{\prime}=M_{i}$ for $i=1,2,3,4, M_{6}^{\prime}=M_{5}, M_{5}^{\prime}=\operatorname{coker}\left(M_{6} \rightarrow\left(M_{5} \perp_{4} M_{5}\right)\right)$; $\alpha_{56}^{\prime} m=\operatorname{class}(0, m), B_{56^{m}}^{\prime}=\operatorname{class}(m, 0), \gamma_{i 5} \operatorname{class}\left(m, m^{\prime}\right)=\gamma_{i 5^{m}}$ $(i=1,2),=r_{i 5^{m}}(i=3 ; 4)$. The functor establishes an equivalence between the full subcategory of $\bmod \left(R_{2}\right)$ whose objects are the $R_{2}$-modules without direct summands isomorphic to the injective module $R_{2} \varepsilon_{6} /\left(\mathrm{rad} R_{2}\right) \varepsilon_{6}$, and the full subcategory of $\bmod \left(R_{2}\right)$ whose objects have no simple quotients isomorphic to $R_{2} \varepsilon_{5} /\left(\operatorname{rad} R_{2}\right) \varepsilon_{5}$. We need, therefore, only classify the indecomposable $R_{2}$-modules in the image of $\Phi$ to obtain a classification of indecomposable modules in $\bmod \left(R_{1}\right)$ and $\bmod \left(R_{2}\right)$.

\section{Matrix regression}

The Nazarova-Roiter regression method of [6] is briefly described here, and will be used later.

A regression scheme $T$ consists of two finite linearly ordered sets $R=\left\{r_{1}, \ldots, r_{m}\right\}, C=\left\{c_{1}, \ldots, c_{n}\right\}$, and a symmetric relation $\rho$ on their disjoint urion which is such that for any $x \in R \Perp C$ there is at most one $y$ related to $i c$.

A $T$-matrix is a block matrix whose block rows are parametrised in order by the set $R^{\prime}$ obtained from $R$ by replacing each $r_{i}$ related to itself by two adjacent elements. The block columns are parametrised in order by the analogous set $c^{\prime}$. If $\rho\left(r_{i}, r_{j}\right)\left(\rho\left(c_{i}, c_{j}\right)\right), i \neq j$, then the corresponding block rows (columns) have the same rumber of rows 
(columns) and if $\rho\left(r_{i}, c_{j}\right)$ the block row has the same number of rows as the number of columns in the block column. Block rows or columns may have zero width.

Two T-matrices are said to be isomorphic if one can be transformed into the other by a sequence of operations of the following types.

1. A block row may be added to any subsequent block row, except that the two block rows corresponding to $r_{i}$ related to itself may not be added to each other.

2. A block row may be multiplied on the left by any invertible matrix, subject to

(a) if $\rho\left(r_{i}, r_{j}\right), i \neq j$, the corresponding block rows may be simultaneously multiplied by the same matrix,

(b) if $\rho\left(r_{i}, c_{j}\right)$, multiplication of the corresponding row on the left by an invertible matrix entails multiplication of the corresponding column on the right by its inverse.

3. The analogues of 1,2 (a) for columns.

The direct sum of two T-matrices is defined in the usual way, and the Krull-Schmidt Theorem applies. Nazarova and Roiter [6] have shown that the indecomposable $T$-matrices are isomorphic either to a discrete canonical $T$-matrix, parametrized by combinatorial invariants independent of the field $K$, or to continuous canonical $T$-matrices parametrized by combinatorial invariants and a monic irreducible polynomial. The proof is a complicated induction for all regression schemes simultaneously.

\section{The four-subspace classification}

Reference [5] is not widely available and it deals only with the case when the field $K$ is algebraically closed. It may therefore be convenient to use Brenner's note [2], which contains the general case including the extension to skew fields, and also gives other references. Indeed, the entire contents of this paper may be extended to the skew field case with only a few small formal changes.

The symbol $\lambda$ refers to an element of $K$ in the case when the field 
is algebraically closed. This is equivalent to a linear monic polynomial: in general the symbol refers to an arbitrary monic irreducible polynomial.

The classification of 4-subspace systems obtained by Gelfand and Ponomarev in [5] will be needed in the next sections. The construction of indecomposable $R_{2}$-modules uses the natural definition of a 4-subspace system as a quintuple of vector spaces $V_{1}, \ldots, V_{5}$ with linear maps $r_{i}: V_{i}+V_{5}$. At the same time it is convenient to think of the submodule of an $R_{2}$ module $M$ obtained by ignoring $M_{6}, \alpha_{56}, \beta_{56}$ as a 4-subspace system ker $\gamma_{i} \rightarrow M_{5}$. If $M$ has no direct summands isomorphic to $R_{2} \varepsilon_{i}$, $i=1, \ldots, 4$, the structure of this 4-subspace submodule is completely determined by the system ker $\gamma_{i}+M_{5}$.

On pages 22, 23 there is a complete list of the isomorphism types of indecomposable 4-subspace systems that will be needed. The dimension of a system is the quintuple $\left(\operatorname{dim} V_{1}, \ldots, \operatorname{dim} v_{5}\right)$.

The dimension of a non-trivial intersection is always $I$. With the exception of $S(2 n, 0, \lambda)$, in each case the system is characterised by its dimension and non-trivial intersections. Gelfand and Ponomarev [5] give bases for the spaces that can be used to calculate the homomorphisms of any system into any other, as also does Brenner [2]. Some information about homomorphisms is needed in Section 5 , and is recorded there.

\section{The representation type of $R_{2}$}

It is logically redundant to discuss the representation type of $R_{2}$, given that Section 5 describes the construction of the indecomposable $R_{2}-$ modules in the image of the functor $\Phi$. Nevertheless, a discussion is given here, on the grounds that the method of obtaining regression schemes to classify $R_{2}$-modules directly may be of some interest.

Let $N$ be any positive integer, and $M$ an $R_{2}$-module whose 4-subspace submodule has indecomposable summands whose components in $M_{5}$ have dimension at most $2 N$. Any direct summand of the 4-subspace 


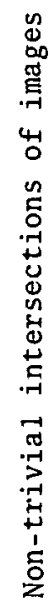

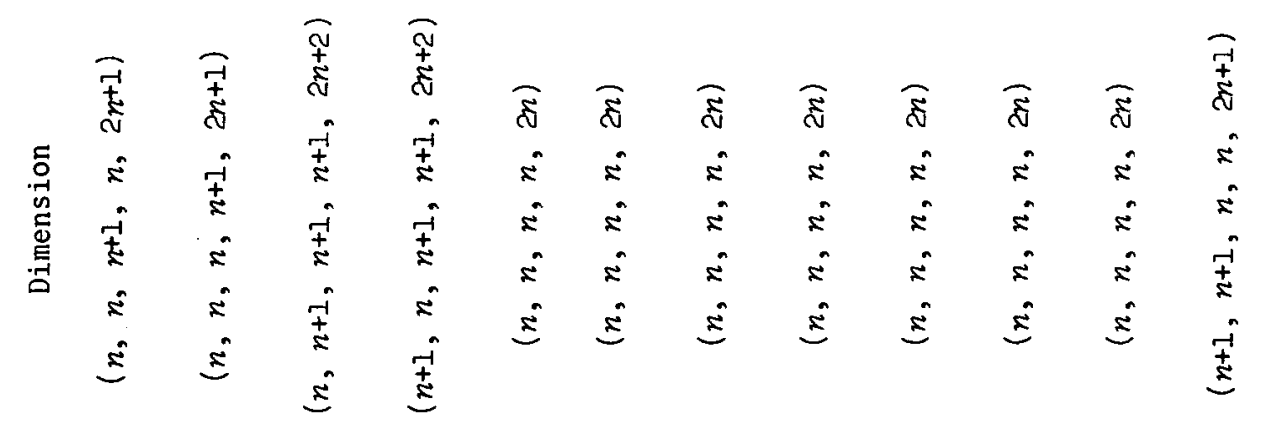

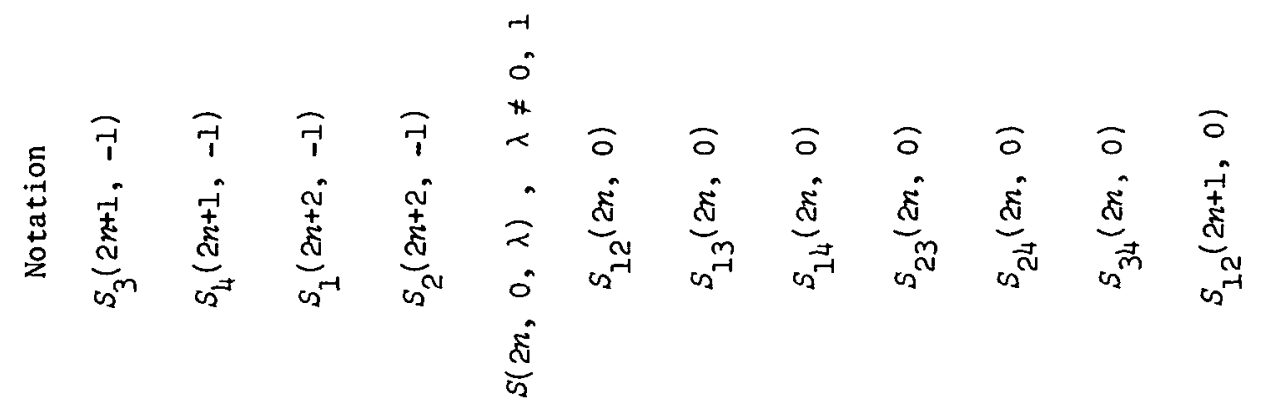




$$
\begin{aligned}
& \text { oิ o } \widehat{0} \text { o } \\
& \wedge \wedge \wedge \wedge
\end{aligned}
$$

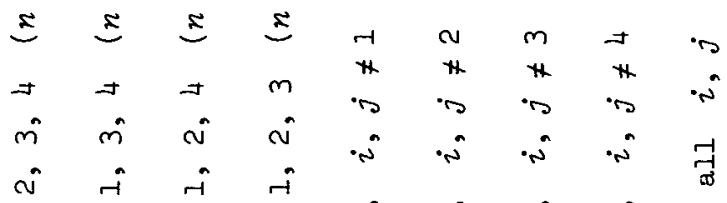

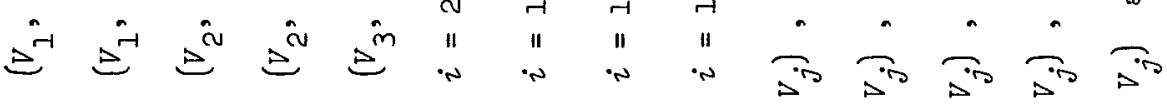

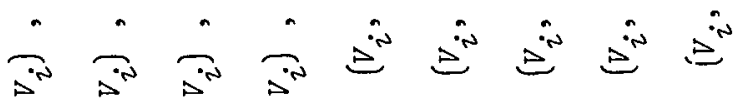

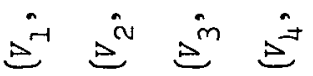

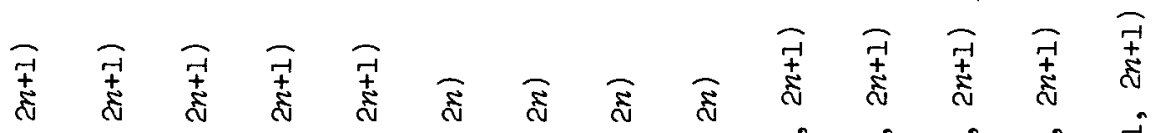

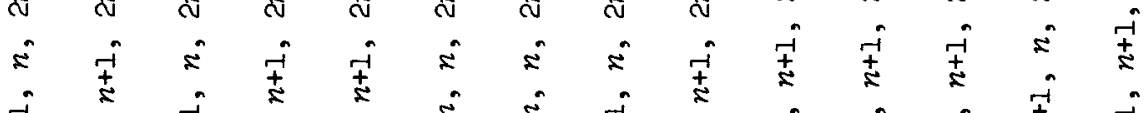

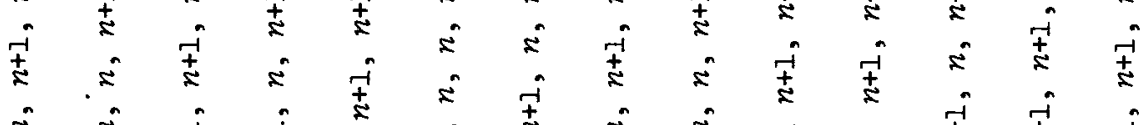

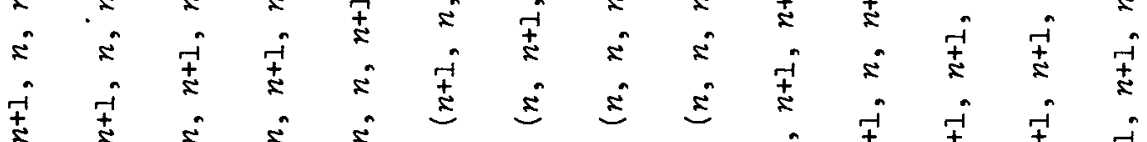

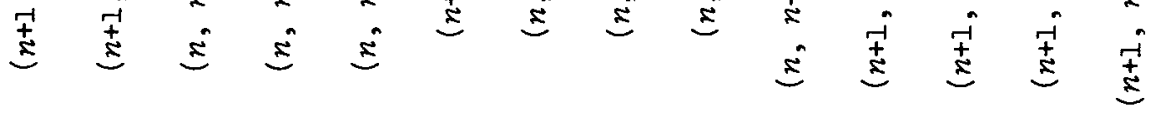

$$
\begin{aligned}
& \text { ○े } 0 \text { 0 } 0 \text { व }
\end{aligned}
$$

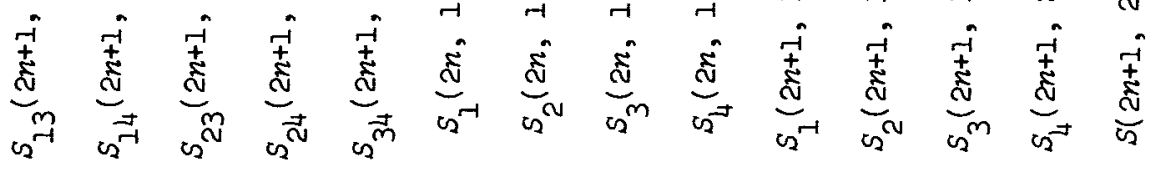


submodule such that $\operatorname{ker} \gamma_{1} \cap \operatorname{ker} \gamma_{2}=0=\operatorname{ker} \gamma_{3} \cap \operatorname{ker} \gamma_{4}$ is obviously a direct summand of $M$, so we shall assume that no such summands occur. The summands then correspond to 4-subspace systems $\operatorname{ker} \gamma_{i} \rightarrow M_{5}$ of types $s(2 n+1,2), \quad s_{i}(m, 1), i=1,2,3,4, S_{12}(m, 0), S_{34}(m, 0)$. One can then define various natural submodules in terms of those summands that appear, as follows:

Submodule

$$
\begin{aligned}
& a(k) \\
& b_{3}(k) \\
& c_{34}(k)
\end{aligned}
$$

\section{4-subspace systems indexing its summands}

The submodules $b_{1}(k), b_{2}(k), b_{4}(k)$ are obtained by applying . permutations $(13)(24),(14)(23),(12)(34)$ to the subscripts in the definition of $b_{3}(k)$, and the submodule $c_{12}(k)$ by applying (13)(24) to those in the definition of $c_{34}(k)$.

There are inclusions as follows:

(1) $\quad a(1) \subset a(2) \subset \ldots \subset a(N)$,

(2) $\quad a(N) \subset b_{1}(1), b_{2}(1) \subset c_{12}(1) \subset b_{1}(2), b_{2}() \subset c_{12}(2) \subset \ldots$,

(3) $\quad a(N) \subset b_{3}(1), b_{4}(1) \subset c_{34}(1) \subset b_{3}(2), b_{4}(2) \subset c_{34}(2) \subset \ldots$.

Now the problem of obtaining simultaneous canonical forms for $\gamma_{15}, \gamma_{25}$, $\gamma_{35}, \gamma_{45}$, and $\beta=B_{56}$, is a problem of finite representation type. This is evident from the fact that im $\beta \subset$ ker $\gamma_{35} \cap$ ker $\gamma_{45} \subset c_{34}(2 N-1)$, and 
from the structure of the filtration of ker $\gamma_{35} \cap$ ker $\gamma_{45}$ induced by (1) and (3). The reader may check from Gelfand and Ponomarev [5] the classification of 3-subspace systems needed to calculate filtration (4) below. This classification may be used to check the naturality of the filtrations. The induced filtration of $M_{6}$ is:

(4) $0 \subset \beta^{-1} a(1) \subset \ldots \subset \beta^{-1} a(N) \subset \beta^{-1} b_{3}(1)$,

$$
\beta^{-1} b_{4}(1) \subset \beta^{-1}\left(b_{3}(1)+b_{4}(1)\right) \subset \ldots,
$$

and it may be used to put $B$ into canonical form. A filtration of $X=\operatorname{ker} \gamma_{15} n \operatorname{ker} \gamma_{25}$ is likewise induced by (1) and (2). There are isomorphisms $B^{-1} a(k+1) / B^{-1} a(k) \rightarrow a(k+1) / a(k)$ for $1 \leq k \leq N-1$. Thus the problem of finding canonical forms for $\alpha_{56}$ compatible with those for $\beta_{56}$ is equivalent to the canonical form problem for the evident regression scheme. As $N$ is arbitrary, this classifies all indecomposable $R_{2}$-modules.

\section{Construction of indecomposable modules}

Let $M \in \underline{\underline{C}}$ where $\underset{\mathrm{C}}{\underline{\mathrm{C}}}$ is the full subcategory of the image of $\Phi$ whose objects have no direct summands isomorphic to any of the simple projective modules $R_{2} \varepsilon_{i} \quad(i=1, \ldots, 4)$. Write $\alpha$ for $\alpha_{56}, \beta$ for $\beta_{56}, \gamma_{i}$ for $\gamma_{i 5}$. Since $M_{5}=\operatorname{ker} \gamma_{1} n \operatorname{ker} \gamma_{2}+\operatorname{ker} \gamma_{3} n \operatorname{ker} \gamma_{4}$, the 4-subspace submodule of $M$ can have direct summands only of the types corresponding to the systems $S_{34}(1,0), S_{1}(1,1), S_{2}(1,1)$, $S_{12}(1,0), S_{3}(1,1), S_{4}(1,1)$, or $S(1,2)$. Decomposition of $M_{5}$ into a direct sum of subspaces $w_{i}, i=1, \ldots, 7$, corresponding to summands of each type (in the given order) allows one to obtain block matrices in canonical form for the $\gamma_{1}, \gamma_{2}, \gamma_{3}, \gamma_{4}$ as follows:

$$
r_{1}\left[\begin{array}{lllllll}
1 & 0 & 0 & 0 & 0 & 0 & 0 \\
0 & 1 & 0 & 0 & 0 & 0 & 0
\end{array}\right] \text {, }
$$




$$
\begin{aligned}
& \gamma_{2}\left[\begin{array}{lllllll}
1 & 0 & 0 & 0 & 0 & 0 & 0 \\
0 & 0 & 1 & 0 & 0 & 0 & 0
\end{array}\right], \\
& \gamma_{3}\left[\begin{array}{lllllll}
0 & 0 & 0 & 1 & 0 & 0 & 0 \\
0 & 0 & 0 & 0 & 1 & 0 & 0
\end{array}\right], \\
& \gamma_{4}\left[\begin{array}{lllllll}
0 & 0 & 0 & 1 & 0 & 0 & 0 \\
0 & 0 & 0 & 0 & 0 & 1 & 0
\end{array}\right] .
\end{aligned}
$$

Given that, for any such $M$, im $\beta \leq W_{1}+W_{2}+W_{3}+W_{7}$, and $W_{1}+W_{2}+W_{3} \leq$ im $\beta+W_{7}$, one can reduce the matrix of $\beta$, using operations compatible with the filtration, to the form on the left below, where the marked divisions correspond to the division of the $\gamma_{i}$ into columns, except that the last block row is further subdivided. The matrix of $\alpha$ is then in the form on the right below with block rows and columns corresponding to those of $B$ :

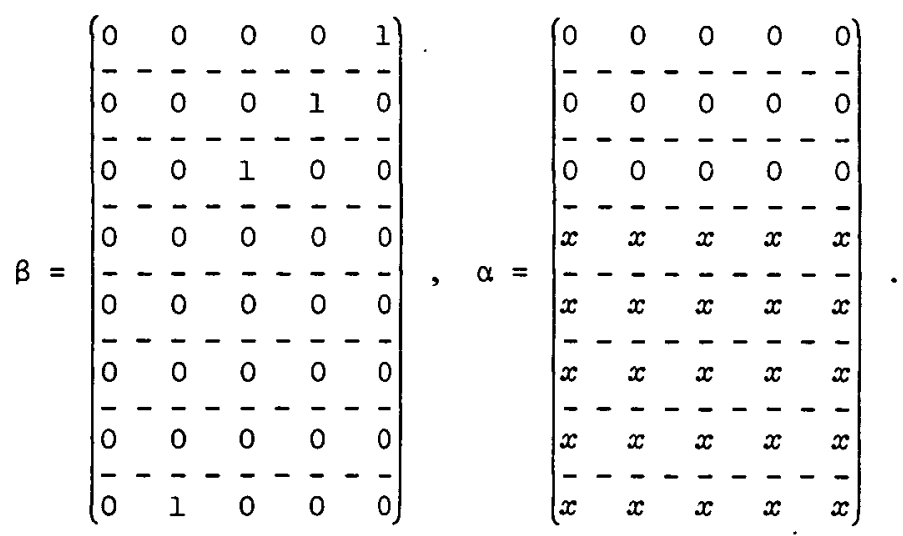

Compatibility requirements imply that the permissible operations on the nonzero part of the matrix of $\alpha$ are those permitted by the following regression scheme:

$$
\begin{aligned}
& R=\left\{r_{1}, r_{2}, r_{3}, r_{4}\right\}, C=\left\{c_{1}, c_{2}, c_{3}, c_{4}\right\}, \\
& \rho=\left\{\left(r_{2}, r_{2}\right),\left(r_{4}, c_{2}\right),\left(c_{2}, r_{4}\right),\left(c_{3}, c_{3}\right)\right\},
\end{aligned}
$$

where the rows and columns of $\alpha$ are grouped and ordered so that 
$r_{1}$ corresponds to block row 4 ,

$r_{2}$ to block rows 5 and 6 ,

$r_{3}$ to block row 7 ,

$r_{4}$ to block row 8 , $c_{1}$ to block column 1 ,

$c_{2}$ to block column 2 ,

$c_{3}$ to block columns 3 and 4 ,

$c_{4}$ to block column 5 .

Note that $M \in \underline{\underline{C}}$ requires that the blocks corresponding to $r_{1}, r_{2}$,

$r_{3}$ must have rank equal to the number of rows they contain.

The canonical forms for the matrices $\alpha$ that produce indecomposable modules in $\underline{\underline{C}}$ are thus obtainable from the rules given by Nazarova and Roiter in [6]. The rules are abstracted from the process of reduction of each component of a $T$-matrix to canonical form, which, at each stage, replaces the regression scheme by a new one with finer divisions of the block rows and columns. A brief reinterpretation of the process, as applicable to modules in $\underline{\underline{C}}$ is given here.

At each stage of the regression, one may regard the block columns (rows) of the matrix of $\alpha$ as being indexed by triples $(M, \phi, Q)$ $((N, \psi, P))$ where $M(N)$ is an indecomposable module with a distinguished quotient $Q$ (submodule $P$ ), and $\phi(\psi)$ is a fixed surjection $M \rightarrow Q$ (injection $P \rightarrow N$ ). Distinct block columns (rows) related by $\rho$ have the same $M(N)$, while a block column related to a block row has $M \simeq N$.

The block column $(M, \phi, Q)$ (row $(N, \psi, P)$ ) is to the left of $\left(M^{\prime}, \phi^{\prime}, Q^{\prime}\right)$ (above $\left(N^{\prime}, \psi^{\prime}, P^{\prime}\right)$ ) if and only if there are homomorphisms $\mu: M^{\prime} \rightarrow M, \kappa: Q^{\prime} \rightarrow Q\left(\nu: N \rightarrow N^{\prime}, \pi: P \rightarrow P^{\prime}\right)$ such that $\kappa(\pi)$ is surjective and $K \phi^{\prime}=\phi \mu \quad\left(\psi^{\prime} \pi=\nu \psi\right)$. Two block columns (rows) correspond to the same (self-related) element of the regression scheme if and only if they are indexed by modules having no such homomorphisms between them in either direction.

The module $Q(P)$ is always generated by $\phi_{6}($ ker $\alpha)$ $\left(\psi_{2}^{-1}\left(\right.\right.$ coker $\alpha \cap$ ker $\gamma_{1} \cap$ ker $\left.\left.\gamma_{2}\right)\right)$ and may always be chosen isomorphic to one of those indexing the columns (rows) in the initial scheme; namely, 


$$
\begin{aligned}
& c_{4}: M_{4}=Q_{4}=R_{2} \varepsilon_{6} /\left\langle\alpha R_{2} \varepsilon_{6}\right\rangle, \\
& c_{3}: M_{3}=Q_{3}=M_{4} /\left\langle\gamma_{2} M_{4}\right\rangle, \\
& \bar{M}_{3}=\bar{Q}_{3}=M_{4} /\left\langle\gamma_{1} M_{4}\right\rangle, \\
& c_{2}: M_{2}=M_{3} /\left\langle\gamma_{1} M_{2}\right\rangle, Q_{2}=M_{2} /\left\langle\beta M_{2}\right\rangle, \\
& c_{1}: M_{1}=Q_{1}=Q_{2}, \\
& r_{1}: N_{1}=P_{1}=\left\langle\alpha R_{2} \varepsilon_{6}\right\rangle, \\
& r_{2}: N_{2}=P_{2}=P_{1} /\left\langle\gamma_{3} P_{1}\right\rangle, \\
& \bar{N}_{2}=\bar{P}_{2}=P_{1} /\left\langle\gamma_{4} P_{1}\right\rangle, \\
& r_{3}: N_{3}=P_{3}=\left\langle\beta M_{2}\right\rangle, \\
& r_{4}: N_{4}=M_{3}, P_{4}=\left\langle\beta M_{2}\right\rangle .
\end{aligned}
$$

Each step of the regression constructs new indecomposable modules, by specifying linear maps from the kernel of $\alpha$ in the column-modules to the cokernel of $\alpha$ in the row-modules in such a way that the kernel of the map contains all copies of $(\operatorname{ker} \phi)_{6}$ (or $(\operatorname{ker} \phi)_{6}$ and $(\operatorname{ker} \bar{\phi})_{6}$ when the column is self-related) and the image of the map is contained in the space spanned by all copies of $(\text { im } \psi)_{5}$ (or $(\text { im } \psi)_{5}$ and $(\text { im } \bar{\psi})_{5}$ when the row is self-related).

If the column is related to the row, the canonical forms for the matrices of maps producing new indecomposable modules are indecomposable rational canonical forms. Self-relation of a column specifies two distinguished subspaces in the domain of the map, and self-relation of the row specifies two in the codomain. When the column is not related to the row, then, the construction corresponds to the construction of certain indecomposable 2-subspace, 3-subspace, or 4-subspace systems, according to the degree of self-relation present. The systems are restricted by the requirement that the subspaces corresponding to the rows span the junction space and must have trivial intersection if there are two of them. Twosubspace constructions are obvious. To identify the 3- and 4-subspace systems that produce new indecomposable modules, we use the 4-subspace notation from section 2, assuming that the first two subspaces correspond 
to columns and the last two to rows, and that, in the case of 3-subspace systems, an appropriate subspace is zero. The type's that occur are then $S_{i j}\{m, 0\},\{i, j\}=\{1,3\},\{1,4\},\{2,3\},\{2,4\} ;$ $S(2 n, 0, \lambda) ; S_{12}(2 n, 0) ; S_{3}(2 n+1,-1), S_{4}(2 n+1,-1) ;$ $S_{1}(2 n,-1), S_{2}(2 n,-1) ; S_{3}(2 n+1,1), S_{4}(2 n+1,1)$; $s_{1}(2 n, 1), s_{2}(2 n, 1)$.

New constructions index new block rows and/or columns if and only if they continue to have distinguished quotients generated by elements of ker $\alpha$ or distinguished submodules generated by elements of coker $\alpha \cap$ ker $\gamma_{1} \cap$ ker $\gamma_{2}$, and the dimensions of these two subspaces indicate how many new index pairs must occur. Thus, for example, when both block row and column used in the construction are self-related, a new construction indexes a new block column if and only if the two subspaces corresponding to columns intersect nontrivially, and indexes a new block row if and only if these two subspaces do not span the junction space.

Relations between, and ordering of the modules indexing new block rows and columns are determined using the rules given above. The special homomorphisms used in ordering are present between new constructions arising from the same block row and column if and only if there are similarly defined special homomorphisms between the subspace systems used in the construction. For example, the new columns obtained from 4subspace systems are ordered, from left to right, as follows: $\left\{S_{3}(2 n-1,1), S_{4}(2 n-1,1)\right\} \quad$ (two block columns corresponding to a selfrelated element)

$$
\begin{aligned}
& \left\{S_{1}(2 n, 1), S_{2}(2 n, 1)\right\} \quad\left\{S_{3}(2 n+1,1), S_{4}(2 n+1,1)\right\} \\
& \ldots s_{12}(2 n+2,0), s_{12}(2 n, 0) \ldots .
\end{aligned}
$$

The new rows obtained are ordered from top to bottom as follows: $\left\{s_{1}(2 n,-1), s_{2}(2 n,-1)\right\}$ (two block rows corresponding to a self-related element)

$$
\begin{aligned}
\left\{S_{3}(2 n+1,-1), S_{4}(2 n+1,-1)\right\} & \left\{S_{1}(2 n+2,-1), S_{2}(2 n+2,-1)\right\} \\
& \ldots S_{12}(2 n+2,0), S_{12}(2 n, 0) \ldots .
\end{aligned}
$$




\section{Dimensions of indecomposable modules}

If $M$ is an $R_{i}$-module, the dimension of $M$ is the sextuple $\left(m_{1}, \ldots, m_{6}\right)$, where $m_{j}$ is the dimension of the vector space $M_{j}$.

The algebras $R_{1}$ and $R_{2}$ are algebras corresponding to quivers with relations, the relations in these two cases stating that certain composites of arrows in the quiver are equal or zero. In [3], Brenner defines the quadratic from $q(x)$ associated with a quiver with relations as

$$
q(x)=\sum_{i=1}^{n} x_{i}^{2}+\sum_{i \neq j} a_{i j} x_{i} x_{j},
$$

where the quiver has $n$ points, and $a_{i j}=-k$ if there are $k$ arrows from $i$ to $j$ in the quiver, and $a_{i j}=+k$ if there are $k$ (independent) relations on composites of arrows connecting $i$ and $j$. For quivers without relations, and also for those quivers with relations whose represcntation type is known, the representation type of the quiver is finite, tame, or wild (see Brenner [3]) according as the quadratic form is positive definite, positive semidefinite, or indefinite on the cone $\left\{x \mid x_{i} \geq 0\right\}$. The dimensions of indecomposable representations of quivers of finite or tame type are precisely the solutions in non-negative integers to $q(x)=1$ (finite type), 0 or 1 (tame type) (see our notes [4]). The heuristic argument provided by Brenner in [3] to explain this, views $q(m)-I$ as measuring the degree to which endomorphisms of a representation of the quiver, in general position, of dimension $m$, are determined, modulo scalars, by the requirements of compatibility with the linear maps in the representation. The functors of Section 1 induce isometries.

The quadratic form for $R_{2}$ is

$$
q(x)=\sum_{i=1}^{4}\left[x_{i}-\frac{1}{2}\left(x_{5}-x_{6}\right)\right]^{2},
$$

which is clearly semidefinite, of co-rank 2. If $x_{1}=x_{2}=x_{3}=x_{4}=0$ it becomes the quadratic form $q_{1}$ associated with the quiver $\hat{A}_{1}$ (the 'Kronecker problem'), and if $x_{6}=0$ it becomes the quadratic form $q_{2}$ 
associated with the quiver $\hat{D}_{4}$ (the 4-subspace problem). The solutions to $q(x)=0$ are of form $n a+m b$, where $a=(0,0,0,0,1,1)$ (corresponding to $q_{1}\left(a^{\prime}\right)=0, a^{\prime}=(1,1)$ ) and $b=(1,1,1,1,2,0)$ (corresponding to $q_{2}\left(b^{\prime}\right)=0, b^{\prime}=(1,1,1,1,2)$ ). The solutions to $q(x)=1$ are of form $n a+m b+r$ where $r$ is either $(0,0,0,0,0,1)$ or one of the 24 values $\left(r_{1}, r_{2}, r_{3}, r_{4}, r_{5}, 0\right)$ with $0 \leq r_{1} \leq 1$, $q(r)=1$.

The construction method of Section 5 , however, reveals the existence of indecomposable $R_{2}$-modules whose dimension $m$ is such that $q(m)=2$. Simple calculations of dimensions arising in the course of construction of indecomposable modules in $\underline{\underline{C}}$, together with the use of the functor $\Phi$ and its adjoint, show that the set of dimensions of indecomposable $A_{2}$-modules splits into three subsets as follows.

(1) Solutions to $q(x)=0$. All dimensions $n a+m b, n, m \geq 0$, are in fact realised as dimensions of indecomposable modules. The infinite families of indecomposable modules of the same dimension, parametrised by the monic irreducible polynomials over the field, are all of this dimension type.

(2) Solutions to $q(x)=1$. Again, the dimensions $n a+m b+r$ are all realized for $n, m \geq 0$.

(3) Solutiors to $q(x)=2$. Those that arise as dimensions of indecomposable modules are of form $n a+m b+s$, where $s$ is one of the following :

$$
\begin{aligned}
& s_{1}=(1,1,0,0,0,0), s_{2}=(0,0,1,1,0,0), \\
& s_{3}=(1,1,0,0,2,0), s_{4}=(0,0,1,1,2,0), \\
& s_{5}=(2,0,1,1,2,0), s_{6}=(0,2,1,1,2,0), \\
& s_{7}=(1,1,2,0,2,0), s_{8}=(1,1,0,2,2,0) .
\end{aligned}
$$

If $s=s_{i}, i=1, \ldots, 4$, the dimension is realised for all choices of $n \geq 1, m \geq 0$, while if $s=s_{i}, i=5, \ldots, 8$, it is realised for all $n \geq 3, m \geq 0$. 


\section{Further extensions of this method}

It should be noted that our notes [4] on the representation theory of the Dynkin diagrams $A_{n}, D_{n}$, and $E_{n}$, and the corresponding extended Dynkin diagrams have been generalised by $D / a b$ and Ringel in numerous papers so as to include the other Dynkin diagrams. It is to be expected that analogous generalisation is possible in the context of commutative quivers.

In the absence of a general representation theory of quivers with commutativity and zero-composite relationships, various examples of such problems are discussed below. All have semidefinite quadratic form.

Firstly, for each positive integer $n$ there is a 'factorised $\hat{A}_{2 n-1}$ ' quiver to consider. It may be obtained from the $R_{1}$-module problem of this paper by addition of the requirements that each of the vector spaces $\varepsilon_{1} M, \ldots, \varepsilon_{6} M$ is graded by the integers taken modulo $n$, that $\gamma_{51}, \gamma_{52}$, $\gamma_{53}, \gamma_{54}, \delta_{16}, \delta_{26}$ respect this grading whilst $\delta_{36}, \delta_{46}$ map the $i$ th graded component to the $(i+1)$ th. As before, this gives rise to the corresponding 'graded $R_{2}$-module' problem, which can be handled by the introduction of 'cyclically graded regression schemes'. This concept is a direct generalisation of that described in Section 2.

Secondly, Butler has pointed out, the algebra $R_{2}$ may be extended by the adjunction of further idempotents $\varepsilon_{7}, \varepsilon_{8}, \ldots$, further generators $\alpha_{67}, \beta_{67}, \alpha_{78}, \beta_{78}, \ldots$ subject to relationships $\alpha_{56}{ }_{67}=\beta_{56} \alpha_{67}=0$, and so on. The modules $M$ for which $\varepsilon_{1} M=\varepsilon_{2} 2^{M}=\varepsilon_{3} M=\varepsilon_{4} M=0$ may be classified by use of the filtration induced by the appropriate 'Brauer graph'. However, for the purposes of this paper, it is more convenient to put the mappings $\alpha_{56}, \alpha_{67}, \alpha_{78}, \ldots$ into simultaneous canonical form. (This is a standard finite representation type problem.) The determination of canonical forms for $\beta_{56}, \beta_{67}, \beta_{78}, \ldots$ is then a regression scheme calculation of a particularly simple type. Furthermore, the general classification may be achieved by an evident reworking of the arguments of this paper.

Thirdly, the quiver underlying the algebra $R_{2}$ may be extended by a 
second 4-subspace system by the adjunction of idempotents $\varepsilon_{7}, \varepsilon_{8}, \varepsilon_{9}, \varepsilon_{0}$ and generators $\gamma_{67}, \gamma_{68}, \gamma_{69}, \gamma_{60}$ subject to additional relationships $\alpha_{56} \gamma_{67}=\alpha_{56} \gamma_{68}=\beta_{56} \gamma_{69}=\beta_{56} \gamma_{60}=0$. The regression schemes defined in Section 2 do not appear to be adequate to determine the representation theory of this quiver. The relevant matrix problem may be obtained by putting $\gamma_{15}, \gamma_{25}, \gamma_{35}, \gamma_{45}$ into the canonical form defined by the filtrations (1), (2), and (3) of Section 4 and simultaneously putting $\gamma_{67}, \gamma_{68}, \gamma_{69}, \gamma_{60}$ into the dual canonical form. The matrix problem so obtained can be described by two regression schemes $T_{\alpha}, T_{\beta}$ in which the last elements of $R_{\alpha}, C_{\alpha}, R_{\beta}, T_{\beta}$ are not related to any elements. The last block row of the $T_{\alpha}$-matrix has the same width as the last block row of the $T_{B}$-matrix. If any operation of type 2 (see Section 2) is performed on one of these last block rows, it must also be performed on the other. Similar conditions hold for the last two block columns. The proof of [6] with slight modifications now shows that this extended algebra has tame representation type.

Fourthly, the above generalisations may be carried out simultaneously to yield still more complicated quivers with zero-composite relations. The above methods suffice to prove that they have tame representation type. Hence sundry associated commutative quivers also have tame representation type.

Fifthly, as Brenner has pointed out, the 4-subspace components of the $R_{2}$ problem considered here may be replaced by $E$ type extended Dynkin diagrams. For example, the algebra

$$
\begin{aligned}
R_{3}=K\left\langle\varepsilon_{1}, \varepsilon_{2}, \varepsilon_{3}, \varepsilon_{4}, \varepsilon_{5}, \varepsilon_{6}, \varepsilon_{7}, \varepsilon_{8}, \alpha_{21}, \alpha_{41}, \alpha_{61},\right. \\
\left.B_{32}, \beta_{54}, \beta_{76}, \gamma_{18}, \gamma_{18} \mid \alpha_{21} \gamma_{18}=\alpha_{41} \delta_{18}=\alpha_{61}\left(\gamma_{18}+\delta_{18}\right)=0\right)
\end{aligned}
$$

is obtained analogously from an $\hat{E}_{6}$ algebra (corresponding to the modules with $\varepsilon_{g} M=0$ ) and a 'Kronecker' algebra. New methods seem necessary to classify the $R_{3}$-modules.

It would be of some interest to have a method for generating all the 
algebras with zero-composite defining relations and with tame representation type.

\section{References}

[1] Maurice Auslander, María Inés Platzeck, Idun Reiten, "Coxeter functors without diagrams", preprint.

[2] Sheila Brenner, "On four subspaces of a vector space", J. AZgebra 29 (1974), 587-599.

[3] Sheila Brenner, "Quivers with commutativity conditions and some phenomenology of forms", Representations of algebras, 29-53 (Proc. Internat. Conf., Ottawa, 1974. Lecture Notes in Mathematics, 488. Springer-Verlag, Berlin, Heidelberg, New York, 1975).

[4] Peter Donovan and Mary Ruth Freislich, "The representation theory of finite graphs and associated algebras" (Carleton Mathematical Lecture Notes, 5, 1973).

[5] I.M. Gel fand and V.A. Ponomarev, "Problems of linear algebra and classification of quadruples of subspaces in a finite-dimensional vector space", Hilbert space operators and operator algebras, 163-237 (Proc. Internat. Conf., Tihany, 1970. Colloq. Math. Soc. János Bolyai, 5. North-Holland, Amsterdam, 1972).

[6] Л.А. Назарова, А.В. Ройтер [L.A. Nazarova and A.V. Roiter], "Об одной эадаче И.М. Гельфанда" [A problem of I.M. Gel'fand], Funkcional. Anal. i Prizožen. 7 (1973), no. 4, 54-69. English translation: Eunctional Anal. Appl. 7 (1973), 299-312.

School of Mathematics,

University of New South Wales, Kensington, New South Wales. 